\title{
Stability of Viscous Shock Wave under Periodic Perturbation for Compressible Navier-Stokes-Korteweg System
}

\author{
Lin Chang ${ }^{1}$ \\ ${ }^{1}$ Beihang University
}

February 23, 2022

\begin{abstract}
In this paper, we study the stability of a viscous shock wave for the isentropic Navier-Stokes-Korteweg equations under spaceperiodic perturbation. It is shown that if the initial perturbation around the shock and the amplitude of the shock are small, then the solution of the N-S-K equations tends to the viscous shock.
\end{abstract}

\section{Hosted file}

nsk-05.pdf available at https://authorea.com/users/461933/articles/557487-stability-ofviscous-shock-wave-under-periodic-perturbation-for-compressible-navier-stokes-kortewegsystem 